\title{
18. MESOZOIC FORAMINIFERA, LEG 30, HOLE 288A AND SITE 289
}

Fouad Y. Michael, Atlantic Richfield Company, Geological Science Group, Dallas, Texas

The zones and subzones used in this report are those proposed by Pessagno $(1967,1969)$. Due to the unfossiliferous nature of more than half of the samples examined and the sparsity of the fauna, the data were not tabulated.

$\mathrm{TA}=$ Total abundance of all planktonic foraminifera in the sample.

$\mathrm{RA}=$ Relative abundance of each taxon.

\section{HOLE 288A}

\section{0-288A-9-1 63-66 cm}

TA: 5

\section{RA}

$4 \quad$ Heterohelix striata (Ehrenberg)

3 Heterohelix sp.

2 Pseudotextularia deformis (Kikoine)

2 Pseudoguembelina sp. aff. P. palpebra Bronnimann and Brown

2 Pseudoguembelina sp. aff. P. cornuta Seiglie

1 Racemiguembelina powelli Smith and Pessagno

5 Globotruncana arca (Cushman)

5 Globotruncana contusa (Cushman)

5 Globotruncana stuarti (De Lapparent)

4 Globotruncana stuartiformis Dalbiez

Biostratigraphic determination: Middle Maestrichtian Globotruncana contusa-stuartiformis Zone, Globotruncana gansseri Subzone.

\section{0-288A-9-3 100-102 cm}

TA: 5

RA

3 Heterohelix striata (Ehrenberg)

2 Heterohelix sp.

1 Globigerinelloides volutus (White)

5 Globotruncana arca (Cushman)

4 Globotruncana contusa (Cushman)

4 Globotruncana stuarti (De Lapparent)

5 Globotruncana stuartiformis Dalbiez

Biostratigraphic determination: Same as above.

\begin{tabular}{|c|}
\hline $30-288 \mathrm{~A}-9-4,100-102 \mathrm{~cm}$ \\
\hline $30-288 A-9-5,100-102 \mathrm{~cm}$ \\
\hline $30-288 \mathrm{~A}-9-6,100-102 \mathrm{~cm}$ \\
\hline
\end{tabular}

In the above three samples the following species were reported almost in identical relative abundance:

\section{RA}

5 Heterohelix striata (Ehrenberg)

3 Heterohelix sp. cf. $H$. palpebra Bronnimann and

Brown

5 Heterohelix sp.

2 Pseudotextularia elegans s. l. (Rzehak)

1 Pseudoguembelina costulata (Cushman)

1 Pseudoguembelina excolata (Cushman)
$1 \quad$ Ventilabrella multicamerata de Klasz

3 Globigerinelloides sp.

5 Globotruncana arca (Cushman)

3 Globotruncana contusa (Cushman)

3 Globotruncana fornicata (Plummer)

$5 \quad$ Globotruncana stuarti (De Lapparent)

5 Globotruncana stuartiformis Dalbiez

Biostratigraphic determination: Middle Maestrichtian Globotruncana contusa-stuartiformis Zone, Globotruncana gansseri Subzone.

30-288A-10-1, 27-29 cm

TA: 3

30-288A-9-1, 63-66 cm

TA: 5

Biostratigraphic determination: Middle Maestrichtian Globotruncana contusa-stuartiformis Zone, Globotruncana gansseri Subzone.

30-288A-9-3, 100-102 cm

TA: 5

30-288A-10-1, 27-29 cm

TA: 3

RA

2 Heterohelix striata (Ehrenberg)

2 Heterohelix sp.

2 Globotruncana arca (Cushman)

1 Globotruncana contusa (Cushman)

3 Globotruncana stuartiformis Dalbiez

Biostratigraphic determination: Same as above.

30-288A-10-1, 111-113 cm

TA: 1

Poorly preserved Globotruncana sp.

30-288A-10-2, 100-102 cm

TA: 0

30-288A-11-1, 116-119 cm

TA: 0

Rare benthonics.

30-288A-11-2, 100-102 cm

TA: 0

30-288A-12-1, 122-125 cm

TA: 0

Rare benthonics.

30-288A-13-1, 125-127 cm TA: 0

30-288A-14-1, 126-128 cm

TA: 4

RA

$1 \quad$ Heterohelix striata (Ehrenberg)

1 Heterohelix sp.

1 Praeglobotruncana sp.

3 Globotruncana arca (Cushman)

5 Globotruncana lapparenti Brotzen

2 Globotruncana sp. cf. G. hilli Pessagno 
Biostratigraphic determination: Early Maestrichtian, Globotruncana fornicata-stuartiformis Zone, Rugotruncana subcircumnodifer Subzone.

\begin{tabular}{ll} 
30-288A-15-1, 130-132 cm & TA: 0 \\
30-288A-15-2, 103-104 cm & TA: 0 \\
$\begin{array}{ll}\text { 30-288A-16-1, } 71-73 \mathrm{~cm} & \text { TA: } 0 \\
\text { Rare benthonics. } & \\
\text { 30-288A-17-1, 133-135 cm } & \text { TA: } 0 \\
\text { 30-288A-20-1, 38-40 cm } & \text { TA: } 3 \\
\text { RA } & \\
2 & \text { Praeglobotruncana turbinata (Reichel) } \\
2 & \text { Praeglobotruncana stephani (Gandolfi) } \\
1 & \text { Rotalipora appenninica } \text { (O. Renz) } \\
2 & \text { Rotalipora cushmani (Morrow) } \\
3 & \text { Rotalipora greenhornensis (Morrow) }\end{array}$ \\
\hline
\end{tabular}

Biostratigraphic determination: Late Cenomanian, Rotalipora s.s. Zone, Rotalipora cushmani-greenhornensis Subzone.

30-288A-20-2, $72-74 \mathrm{~cm}$
Few broken, recrystallized Hedbergella $\mathrm{sp} . ?$

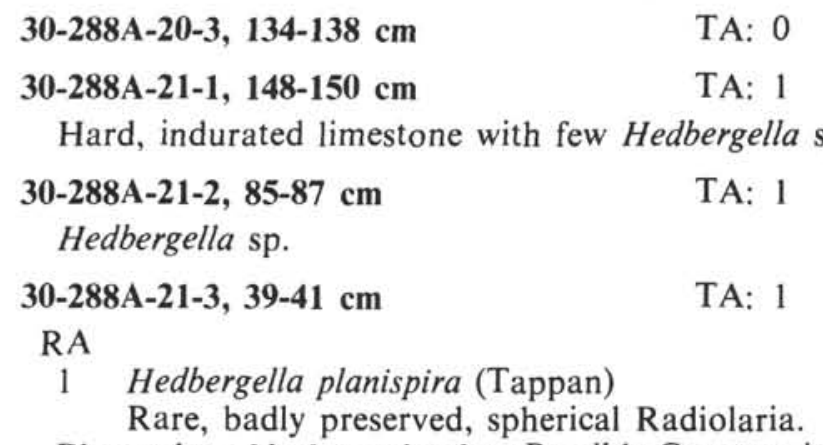

Biostratigraphic determination: Possibly Cenomanian.

Due to the difficulty of isolating foraminifera from the following three samples, they were examined in thin sections. Although the thin sections showed fossiliferous limemud, it was not possible to identify any of the foraminifera to the species level.

\section{0-288A-22-2, 91-93 cm}

Globigerina-type forms and rounded Radiolaria are abundant and recrystallized.

\section{0-288A-23-1, 80-82 cm}

In thin section some keeled forms were recognized, Rotalipora sp.

\section{0-288A-23-2, 2-4 cm}

This section showed Hedbergella sp. and doubtful recrystallized keeled forms.
30-288A-23-3, $77-79 \mathrm{~cm}$
TA: 0
30-288A-24-1, 73-75 cm
TA: 0
30-288A-25-1, 91-92 cm
TA: 1

Small, badly preserved Hedbergella sp. and few spherical radiolarian $\mathrm{sp}$.

\section{0-288A-26-1, 121-123 cm}

In thin section: fossiliferous limemud with recrystallized sponge spicules and Radiolaria.

\section{0-288A-27-1, 122-126 cm}

In this section: few recrystallized, badly preserved Radiolaria and Hedbergella-type forms.

\section{0-288A-27-2, 100-102 cm TA: 1}

Small, badly preserved Hedbergella sp. ?

\section{0-288A-28-1, 42-44 cm}

In thin section: few Hedbergella-type forms with frequent recrystallized Radiolaria and sponge spicules.

30-288A-29-1, 107-109 cm

In thin section: badly crystallized Hedbergella-type forms with rare benthonics and Radiolaria.

Biostratigraphic determination: Core 20, Section 2, through Core 29, Section 1, no specific age can be assigned. Via superposition these cores are pre-late Cenomanian, possibly no older than Albian.

30-288A-30-1, 96-97 cm

TA: 0

\section{SITE 289}

30-289-122-1, 119-121 cm

TA: 0

30-289-122-2, 46-48 cm

TA: 5

RA

$4 \quad$ Heterohelix striata (Ehrenberg)

3 Heterohelix sp.

$2 \quad$ Pseudotextularia sp. cf. P. elegans (Rzehak)

2 Pseudoguembelina excolata (Cushman)

2 Pseudoguembelina sp. cf. P. palpebra Bronnimann and Brown

1 Racemiguembelina sp. cf. $R$. powelli Smith and Pessagno

5 Globotruncana arca (Cushman)

5 Globotruncana contusa (Cushman)

1 Globotruncana stuarti (De Lapparent)

5 Globotruncana stuartiformis Dalbiez

Biostratigraphic determination: Middle Maestrichtian, Globotruncana contusa-stuartiformis Zone, Globotruncana gansseri Subzone.

30-289-123-1, 129-130 cm

TA: 3

30-289-124-1, 123-125 cm

TA: 3

The following species were recognized, almost in the same abundance, in the above two samples.

RA

1 Heterohelix striata (Ehrenberg)

1 Heterohelix sp.

1 Pseudoguembelina sp.

4 Globotruncana arca (Cushman)

3 Globotruncana contusa (Cushman)

1 Globotruncana stuarti (De Lapparent)

3 Globotruncana stuartiformis Dalbiez

Biostratigraphic determination: Same as above. 
TA: 4

RA

2 Heterohelix striata (Ehrenberg)

2 Heterohelix sp.

1 Pseudoguembelina sp.

4 Globotruncana arca (Cushman)

3 Globotruncana contusa (Cushman)

1 Globotruncana stuarti (De Lapparent)

3 Globotruncana stuartiformis Dalbiez

3 Globotruncana sp.

Biostratigraphic determination: Same as above.

30-289-125-1, 142-144 cm

TA: 5

RA

$5 \quad$ Heterohelix striata (Ehrenberg)

5 Heterohelix sp.

1 Pseudotextularia elegans (Rzehak)

1 Pseudoguembelina excolata (Cushman)

1 Pseudoguembelina sp. cf. P. kempensis Esker

1 Pseudoguembelina sp. cf. P. palpebra Bronnimann and Brown

1 Racemiguembelina sp. cf. $R$. powelli Smith and Pessagno

5 Globotruncana arca (Cushman)

4 Globotruncana contusa (Cushman)

1 Globotruncana stuarti (De Lapparent)

5 Globotruncana stuartiformis Dalbiez

Biostratigraphic determination: Middle Maestrichtian, Globotruncana contusa-stuartiformis Zone, Globotruncana gansseri Subzone.

$\begin{array}{ll}30-289-126-1,140-142 \mathrm{~cm} & \text { TA: } 0 \\ 30-289-127-1,129-131 \mathrm{~cm} & \text { TA: } 0 \\ 30-289-128-1,122-124 \mathrm{~cm} & \text { TA: } 0 \\ 30-289-129-1,128-130 \mathrm{~cm} & \text { TA: } 5\end{array}$

Badly preserved fauna, mainly Heterohelicids. RA

$5 \quad$ Heterohelix striata (Ehrenberg)

5 Heterohelix sp.

5 Pseudoguembelina costulata (Cushman)

$5 \quad$ Pseudoguembelina excolata (Cushman)
3 Globigerinelloides volutus (White)

5 Globigerinelloides sp.

2 Globotruncana arca (Cushman)

2 Globotruncana sp.

Biostratigraphic determination: Early Maestrichtian to late Campanian.

\section{SUMMARY}

Samples from Hole 288A, Cores 9 and 10 (Section 1 only), contain fauna of middle Maestrichtian age, Globotruncana gansseri Zone. Cores 10 through 13 are almost barren. Core 14, Section 1, contains fauna of early Maestrichtian age. Cores 15, 16, and 17 are barren. Core 20, Section 1, is rich in late Cenomanian fauna of the Rotalipora cushmani-greenhormensis Subzone. Cores 21 to 30 are barren in most parts. The few fossiliferous samples are hard and indurated. No isolated foraminifera were obtained through processing. In this sections some keeled forms and Hedbergella sp. were recognized. No precise age can be assigned to this part of the section. However, via superposition, these cores possibly range in age from Cenomanian to Albian.

Samples from Site 289, Cores 122 (Section 2) through 125, contain fauna of middle Maestrichtian age, Globotruncana contusa-stuartiformis Zone, Globotruncana gansseri Subzone. The assemblages are very similar to those reported in Cores 9 and 10 of Site 288A. Cores 126 to 128 are barren. Core 129 , Section 1, contains a poorly preserved fauna of predominant Heterohelicids. On the basis of negative evidence (e.g., absence of Globotruncana contusa) it is possibly of early Maestrichtian to latest Campanian.

\section{REFERENCES}

Pessagno, E.A., Jr., 1967. Upper Cretaceous foraminifera from the Western Gulf Coastal Plain: Paleontogr. Am., v. 5, p. $245-445$.

1969. Upper Cretaceous stratigraphy of the Western Gulf Coast are of Mexico, Texas and Arkansas: Geol. Soc. Am. Mem. 111, p. 1-139. 